\title{
Acoustic Scene Classifier Based on Gaussian Mixture Model in the Concept Drift Situation
}

\author{
Ibnu Daqiqil Id*, Masanobu Abe, Sunao Hara \\ Graduate School of Interdisciplinary Science and Engineering in Health Systems, Okayama University, Okayama, 700-0001, Japan
}

\begin{tabular}{l} 
A R T I C L E I N F O \\
\hline Article history: \\
Received: 15 July, 2021 \\
Accepted: 22 September, 2021 \\
Online: 30 September, 2021
\end{tabular}

Keywords:
Acoustic Scene Classifier
Concept Drift
Kernel Density Estimation
Gaussian Mixture Model

\begin{abstract}
A B S T R A C T
The data distribution used in model training is assumed to be similar with that when the model is applied. However, in some applications, data distributions may change over time. This situation is called the concept drift, which might decrease the model performance because the model is trained and evaluated in different distributions. To solve this problem for scene audio classification, this study proposes the kernel density drift detection (KD3) algorithm to detect the concept drift and the combine-merge Gaussian mixture model (CMGMM) algorithm to adapt to the concept drift. The strength of the CMGMM algorithm is its ability to perform adaptation and continuously learn from stream data with a local replacement strategy that enables it to preserve previously learned knowledge and avoid catastrophic forgetting. KD3 plays an essential role in detecting the concept drift and supplying adaptation data to the CMGMM. Their performance is evaluated for four types of concept drift with three systematically generated scenarios. The CMGMM is evaluated with and without the concept drift detector. In summary, the combination of the CMGMM and KD3 outperforms two of four other combination methods and shows its best performance at a recurring concept drift.
\end{abstract}

\section{Introduction}

Human-computer interaction through audition requires devices to recognize the environment using acoustic sound analysis. One of the primary research topics in this area is acoustic scene classification (ASC), which attempts to classify digital audio signals into mutually exclusive scene categories. ASC is an important area of study covering various applications, including smart homes, context-aware audio services, security surveillance, mobile robot navigation, and wildlife monitoring in natural habitats. Machine audition applications have a high potential to lead to more innovative context-aware services.

We intend to develop an ASC system for environmental or scene audio in specific locations (i.e., beach, shop, bus station, and airport) with different acoustic characteristics. The scene audio contains an ensemble of background and foreground sounds. One of the most important aspects of the audio scene in real life is the concept drift [1], whose data distribution might evolve or change in the future. For example, the foreground event sounds in a bus station, such as an ambulance siren, wind noise, and rustling sounds, might change because of the physical environment, human activities, or nature [2]. These changes cause the acoustic data

*Corresponding Author: Ibnu Daqiqil Id, Okayama, Japan, daqiqil@s.okayamau.ac.jp

www.astesj.com

https://dx.doi.org/10.25046/aj060519 distributions to change, potentially causing a lower performance in the trained model [3].

The simplest solutions for handling the abovementioned problems to maintain the model performance are periodic retraining and redeployment of the model. Nevertheless, these solutions can be time consuming and costly. Moreover, the decision for the frequency of retraining and redeployment is a difficult task. Another promising approach is to use an evolving or incremental learning method [4], [5], where the model is updated when a new subset of data arrives [6]. Each iteration is considered as an incremental step toward revisiting the current model.

In this study, we propose a combine-merge Gaussian mixture model (CMGMM) and kernel density drift detection (KD3) to solve the concept drift problem [7]. The CMGMM is an algorithm based on the Gaussian mixture model (GMM) that adapts to the concept drift by adding or modifying its components to accommodate the emerging concept drift. The algorithm's advantages are adaptation and continuous learning from stream data with a local replacement strategy to preserve previously learned knowledge and avoid catastrophic forgetting.

In [7], we compared the CMGMM to the incremental GMM (IGMM) [8] and KD3 to adaptive windowing (ADWIN) [9] and HDMM [10] in two approaches, namely the active and passive 
approaches. In the active approach, the concept drift is detected using a specific algorithm, then adapts the model. In the passive approach, the model is continuously adapted at a specific fixed interval. The result is that the combination of CMGMM and KD3 outperforms other combinations in two of three evaluation scenarios.

The work described herein extends and improves that of previous publications $[7,11]$ in the following respects:

- The algorithm has been modified to use component pruning to overcome the overfitting problem and support the ScikitMultiflow Framework [12].

- The KD3 hyperparameter is optimized, and the algorithm is evaluated using prequential evaluation for better results and online performance monitoring in several concept drift types and scenarios.

The rest of this paper is organized as follows: Section 2 presents the related work of this research and the fundamental equations used in our proposed solutions; Section 3 describes the proposed CMGMM and KD3; Section 4 outlines the experimental setup; Section 5 discusses the experimental results; and finally, Section 6 provides our conclusions.

\section{Related Work}

This section provides a brief overview of the related work on the concept drift and the fundamental theory used in the proposed method.

\subsection{Concept Drift in Audio Scenes}

A concept is defined as a set of object instances [13]. Probabilistically, a concept is defined using prior class probabilities $p(y)$ and class conditional probabilities $p(X \mid y)$ [4]. $p(y)$ and $p(X \mid y)$ determine the joint distribution $p(X, y)$ [3]; hence, a concept is defined as the joint probability distribution of a set of input features $X$ and the corresponding label $y$ in dataset $\mathfrak{D}$. In this paper, $X$ is an acoustic scene sound defined as a mixture of specific event sounds $(\hat{x})$ perceived and defined by humans [14], and $y$ is the label of $X . X$ has numerous types of acoustic event sounds and background noises $\hat{x}$ that often overlap with each other. In other words, the relationship between of $X$ and $\hat{x}$ determines $p(X, y), X \in\left(\hat{x}_{1}, \hat{x}_{2}, \hat{x}_{3}, . \hat{x}_{i}\right)$, where $i$ denotes the number of $\hat{x}$ in $X$.

In the future, the relationship of $\hat{x}$ in $X$ might change, which then changes the relationship of $p(X, y)$. For example, another event sound might appear, or some existing event sounds may disappear. This situation is called the concept drift, which is expressed as follows:

$$
\exists X: p_{w_{0}}(X, y) \neq p_{w_{n}}(X, y) .
$$

Eq. (1) and Figure 1 describe concept drift as the change in the joint probability distribution between two-time windows, $w_{0}$ and $w_{n}$. Models built on previous data at $w_{0}$ might not be suitable for predicting new incoming data at $w_{n}$. This change may be caused by a change not only in the number of $\hat{x}$, but also in the underlying data distribution of $\hat{x}$. These changes require model adaptation because the model's error may no longer be acceptable with the new data distribution [15].

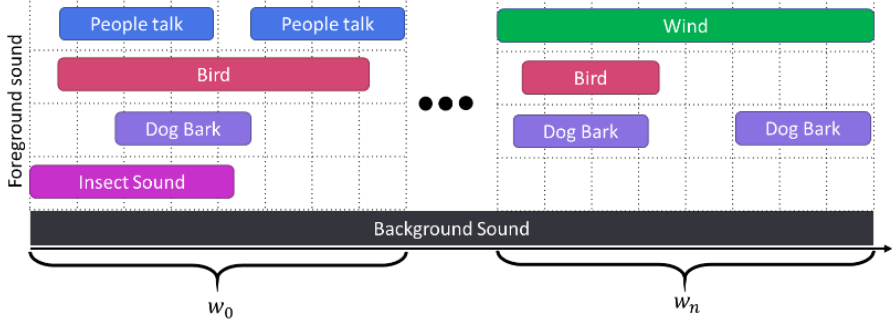

Figure 1: Illustration of the concept drifts in an acoustic scene audio at a park

The change in the incoming data at $w_{n}$ depends on a variety of different internal or external influences (e.g., event sounds that exist in a park depending on the season). The initial data recorded in the winter may only consist of people talking, bird calls, and dogs barking. However, the event sounds change in the summer, and new event sounds, such as insect and wind sounds, emerge.

\subsection{Concept Drift Detection Methods}

Several methods have been proposed to detect concept drifts from a data stream. This study focuses on window-based methods that use fixed windows as a reference for summarizing previous information. This approach has more accurate results than other more straightforward methods, such as cumulative sum [16]. However, the computational time and space used are higher [17]. This approach usually utilizes statistical tests or mathematical inequalities to compute the change in data. Some of the state-ofthe-art methods used in this paper is presented below:

- ADWIN is a sliding window-based concept drift detection algorithm. The size of ADWIN windows $S W$ might change depending on the instance in the distribution. Its size increases when the instance in the stream continues in the same distribution. $S W$ shrinks when distribution changes occur [9]. ADWIN detects concept drifts when the averages between these windows are higher than a given threshold.

- The HDDM is a concept drift detection algorithm based on fixed windows and probability inequalities [10]. The author proposes two types of HDDM, namely HDDMA and HDDMW. The HDDMA uses moving averages, whereas the HDDMW uses weighted moving averages to detect the concept drift. The HDDMA is suitable for detecting the abrupt concept drift, whereas the HDDMW is suitable for detecting the gradual concept drift.

- KSWIN [18] is a window-based concept drift detection method that utilizes the Kolmogorov-Smirnov statistic test (KS-Test) to compare the distances of two distributions. This test is a nonparametric test that does not require any assumptions about the underlying data distribution.

Each method has its optimal hyperparameters, which differ based on the datasets used and the type of drift in those datasets.

\subsection{Merging Gaussian Mixture Component}

A component of a Gaussian distribution is represented by $(w, \mu, P)$ and $\left\{\left(w_{1}, \mu_{1}, P_{1}\right),\left(w_{2}, \mu_{2}, P_{2}\right), \ldots\left(w_{n}, \mu_{n}, P_{n}\right)\right\}$ to denote a mixture of $n$ Gaussian components, where $w, \mu$, and $P$ are the weight or prior probability, distribution means, and covariance matrix, respectively. This mixture must satisfy $w_{1}+w_{2}+\cdots+$ $w_{n}=1$ and has the pdf defined in Eq. (2). 


$$
f(X)=\sum_{i=1}^{n} w_{i} N\left(x ; \mu_{i}, P_{i}\right)
$$

where,

$$
N\left(x ; \mu_{i}, P_{i}\right)=\frac{1}{\sqrt{(2 \pi)^{d} \operatorname{det} P_{i}}} e^{\left[-\frac{1}{2}\left(x-\mu_{i}\right)^{T} P_{i}^{-1}\left(x-\mu_{i}\right)\right]} .
$$

Suppose we wish to merge two Gaussian components $\left\{\left(w_{i}, \mu_{i}, P_{i}\right),\left(w_{j}, \mu_{j}, P_{j}\right)\right\}$, where $w_{i}+w_{j} \leq 1$, and approximate the result as a single Gaussian. The new Gaussian candidate $\left(w_{i j}, \mu_{i j}, P_{i j}\right)$ must preserve the zeroth-, first-, and second-order moments of the original Gaussian. The moment-preserving merge is shown in Eqs. (4)-(6).

$$
\begin{gathered}
w_{i j}=w_{i}+w_{j} \\
\mu_{i j}=\frac{w_{i}}{w_{i}+w_{j}} \mu_{i}+\frac{w_{j}}{w_{i}+w_{j}} \mu_{j} \\
P_{i j}=\frac{w_{i}}{w_{i}+w_{j}} P_{i}+\frac{w_{j}}{w_{i}+w_{j}} P_{j} \\
+\frac{w_{i} w_{j}}{\left(w_{i}+w_{j}\right)^{2}}\left(\mu_{i}-\mu_{j}\right)\left(\mu_{i}-\mu_{j}\right)^{T}
\end{gathered}
$$

\subsection{Kullback-Leibler (KL) Dissimilarity}

Kullback-Leibler (KL) discrimination, known as KL divergence or relative entropy, is a tool for measuring the discrepancy between two probability distributions. The KL discrimination between $f(x)$, a probability distribution for random variables $X$ and $g(x)$, another probability distribution is the expected value of the log-likelihood ratio. The KL divergence of $f(x)$ and $g(x)$ is defined in Eq. (7), where $\Re^{d}$ is the sample space of the random variable $X$.

$$
d_{K L}(f, g)=\int_{\Re^{d}} f(x) \log \frac{f(x)}{g(x)} d x .
$$

Based on Eq. (7), KL is not a perfect distance metric because it is asymmetric $d_{K L}(f, g) \neq d_{K L}(g, f)$ and does not satisfy the triangle inequality, $d_{K L}(f, g)+d_{K L}(g, h) \geq d_{K L}(f, h)$. However, we can use it as metric distance because the KL discrimination of two probability distributions is larger than zero, $d_{K L}(f, g) \geq 0$, and the discrimination of the identical two probability distributions is zero, $d_{K L}(f, f)=0$.

Accordingly, we apply the KL dissimilarity by computing the Kullback-Leibler discrimination upper bound of the post-merge mixture with respect to the pre-merge mixture. In the case of the Gaussian mixture, where $f(x)=N\left(w_{1}, \mu_{1}, P_{1}\right), g(x)=$ $N\left(w_{2}, \mu_{2}, P_{2}\right)$ and $w_{1}+w_{2}<1$, the KL dissimilarity between $f(x)$ and $g(x)$ is shown in Eq. (8).

$$
\begin{aligned}
d_{K L}\left(\left\{\left(w_{1}, \mu_{1}, P_{1}\right)\right\}\right. & \left.,\left\{\left(w_{2}, \mu_{2}, P_{2}\right)\right\}\right) . \\
& =\frac{1}{2}\left[\operatorname { t r } \left(P _ { 2 } ^ { - 1 } \left[P_{1}-P_{2}\right.\right.\right. \\
& \left.\left.+\left(\mu_{1}-\mu_{2}\right)\left(\mu_{1}-\mu_{2}\right)^{T}\right]\right) \\
& \left.+\log \frac{\operatorname{det}\left(P_{2}\right)}{\operatorname{det}\left(P_{1}\right)}\right]
\end{aligned}
$$

${ }^{1}$ https://github.com/ibnudaqiqil/CMGMM www.astesj.com
Please refer to [19] for more details about the KL dissimilarity of the Gaussian distributions.

\section{Proposed Method}

This study extends the combine-merge Gaussian mixture model (CMGMM) [7] to classify audio scenes in concept drift situations. The CMGMM was developed based on the GMM algorithm that can incrementally adapt to the new identified component. This algorithm can add new components as new concepts and update existing components as a response to the change of the current existing concept in the current data. The CMGMM implementation is available in our public repository ${ }^{1}$, with the algorithm pipeline shown in Figure 2.

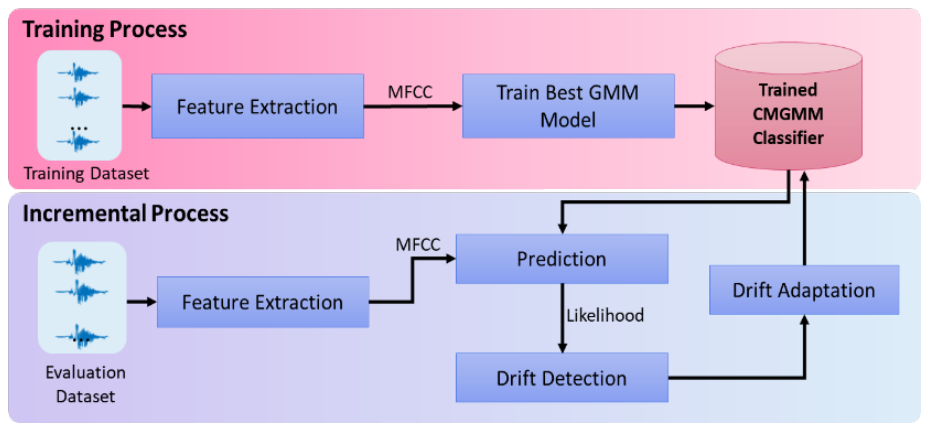

Figure 2: Combine-merge Gaussian mixture model (CMGMM) general workflow

In the training process, we extract the feature of the scene audio from the training dataset $\mathrm{D}_{0}$ and train an optimal model $\mathrm{M}_{\text {optimal }}$. We use the Expectation maximization (EM) [20] algorithm to train the model and the Bayesian information criterion (BIC) [21] to select the best model.

In the incremental process, the $\mathrm{M}_{\text {optimal }}$ performance is observed through the prediction likelihood. When KD3 detects a significant likelihood change, the model activates the concept drift adaptation process. The concept drift adaptation process then begins by creating a local model $\mathrm{M}_{\text {drifted }}$ from the new coming data. $\mathrm{M}_{\text {drifted }}$ represents the new concepts or concept updates in the incoming data. Finally, we combine the $\mathrm{M}_{\text {optimal }}$ and $\mathrm{M}_{\text {drifted }}$ components to include any new concepts from $\mathrm{M}_{\text {drifted }}$ that may not exist in the $\mathrm{M}_{\text {optimal }}$ at the initial training and merge similar components to update the existing component in $\mathbf{M}_{\text {optimal }}$.

The CMGMM pipeline process is detailed in the subsections that follow.

\subsection{Feature Extraction}

Feature extraction is the first step of both the training and incremental processes. In this research, we use normalized Melfrequency cepstral coefficients (MFCCs) that represent the shortterm power spectrum of audio in the frequency domain of the Mel scale. MFCCs are commonly used as features in audio processing and speech recognition. The first step is pre-emphasis for enhancing the quantity of energy in high frequencies. The next step is windowing the signal and computing the fast Fourier transformation to transform the sample from the time domain to the frequency domain. Subsequently, the frequencies are wrapped 
on a Mel scale, and the inverse DCT is applied [22]. Finally, each of the MFCCs is normalized using mean and variance normalization based on Eq. (9):

$$
M F C C_{\text {norm }}=\frac{(M F C C-\mu)}{S},
$$

where, $\mu$ and $S$ denote the mean and the standard deviation of the training samples, respectively.

\subsection{Model Training}

The training process is intended to build a set of models from the training dataset $D_{0}$ containing training data $x=$ $\left\{x_{1}, x_{2}, \ldots, x_{n}\right\}$, where $x_{n}$ denotes the MFCC vector. The models are trained $Q$ times using the EM algorithm. For each training cycle, a different number of components $K$ ranging from $K_{\min }$ to $K_{\max }$ is used, where $Q=\mathrm{K}_{\max }-\mathrm{K}_{\min }$. Consequently, a set of models $\mathcal{M}=\left\{\mathcal{M}_{1}, \mathcal{M}_{2}, \mathcal{M}_{3}, ., \mathcal{M}_{Q}\right\}$ is obtained based on the different numbers of components.

The next step is model selection using the BIC. In [23], the BIC value of a model $\mathcal{M}_{K}$ trained over the dataset $\mathrm{X}$ with $K$ components, $\mathrm{BIC}\left(\mathrm{X}, M_{K}\right)$, is defined as follows:

$$
B I C\left(X, M_{k}\right) \equiv-2 \log L\left(X, M_{K}\right)+v \log N,
$$

where, $L$ denotes the model likelihood; $v$ denotes the degree of freedom of the model parameters; and $N$ denotes the number of training data points. The model with the lowest BIC value is selected because it maximizes the log-likelihood [6]. Algorithm 1 presents the steps of the learning process.

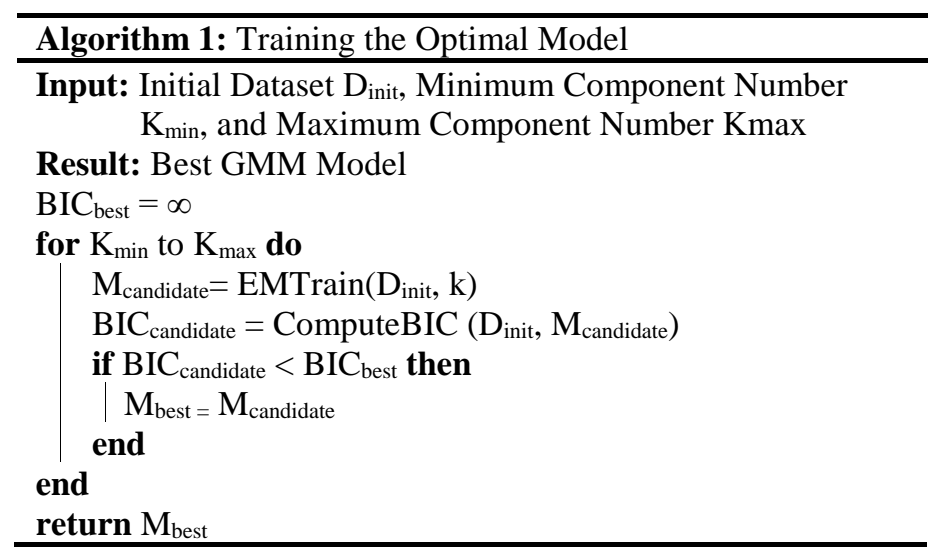

\subsection{Concept Drift Detection}

We propose Kernel Density Drift Detection (KD3) to detect the concept drift. KD3 is a window-based algorithm for concept drift detection. It works based on estimating the window density using the Kernel Density Estimation (KDE) or the Parzen's window [24]. The KDE is a non-parametric probability density estimator that automatically estimates the shape of the data density without assuming the underlying distribution. The concept drift can be detected by comparing the probability functions between these windows. The greater the variation between the windows, the more evidence obtained for the concept drifts. Aside from detecting concept drifts, KD3 also collects data for adaptation $\left(\mathrm{D}_{\text {drift }}\right)$ by identifying a warning zone when data begin to show indications of concept drift.

KD3 requires three hyperparameters, namely $\alpha, \beta$, and $h$, which denote the margins for detecting the concept drift and www.astesj.com accumulating the density distance and the window length, respectively. $\alpha$ is used to determine the threshold of the density variation in the concept drift, while $\beta$ is employed to determine the threshold of the density variation in the warning zone. Therefore, $\alpha$ must be greater than $\beta$. KD3 accepts a set of likelihood windows $z_{c}$ as input. $z_{c}$ is the current likelihood window that contains a sequence of log-likelihood $\ell$ from the model prediction, $z_{c}=$ $\left\{\ell_{1}, \ell_{2}, \ell_{3}, \ldots, \ell_{h}\right\}$.

First, this algorithm aims to estimate the density $\left(f_{k d e}\right)$ of the current $z_{c}$ and previous $z_{c-1}$ windows. Let $\ell_{n}$ be the latest generated $\ell$. Let $z_{c}$ contain the $h$-latest $\ell$ from $\ell_{n}, z_{c} \in$ $\left[\ell_{n-h}, \ell_{n}\right]$, and let $z_{c-1}$ contain the $h$-latest $\ell$ from $\ell_{n-h}, z_{c-1} \in$ $\left[\ell_{n-2 h}, \ell_{n-h}\right]$. To detect a concept drift, the distance $\grave{d}_{t}$ between $f_{k d e}$ of $z_{c}$ and $z_{c-1}$ is computed using Eq. (11) within the bounds of $b 1$ and $b 2$. The bounds are computed based on the maximum and minimum values of the joined $\ell$ of $z_{c}$ and $z_{c-1}$.

$$
\begin{gathered}
\grave{d}_{t}=\frac{1}{2} \int_{b 1}^{b 2}\left|f_{k d e}\left(z_{c}\right)-f_{k d e}\left(z_{c-1}\right)\right| d z, \text { where } \\
z_{c} \in\left[\ell_{n-h}, \ell_{n}\right], z_{c-1} \in\left[\ell_{n-2 h}, \ell_{n-h}\right], \\
b 1=\min \left(\ell_{n-2 h}, \ell_{n}\right), b 2=\max \left(\ell_{n-2 h}, \ell_{n}\right) .
\end{gathered}
$$

Finally, the algorithm compares $\grave{d}_{t}$ to $\alpha$ and $\beta$.hen $\grave{d}_{t}>\beta$. Suppose that the accumulative distance is equal to or greater than $\alpha$. In that case, the algorithm sends the collected data to the model for adaptation. Figure 3 and Algorithm 2 illustrate the detailed KD3 process.

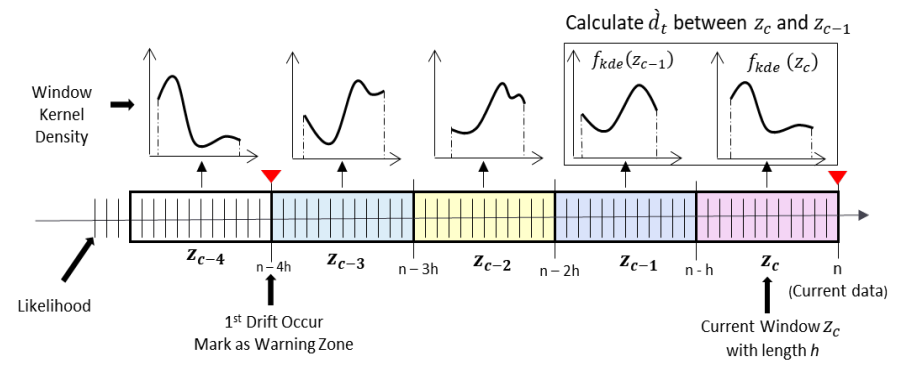

Figure 3: Illustration of the Kernel density drift detection (KD3) concept

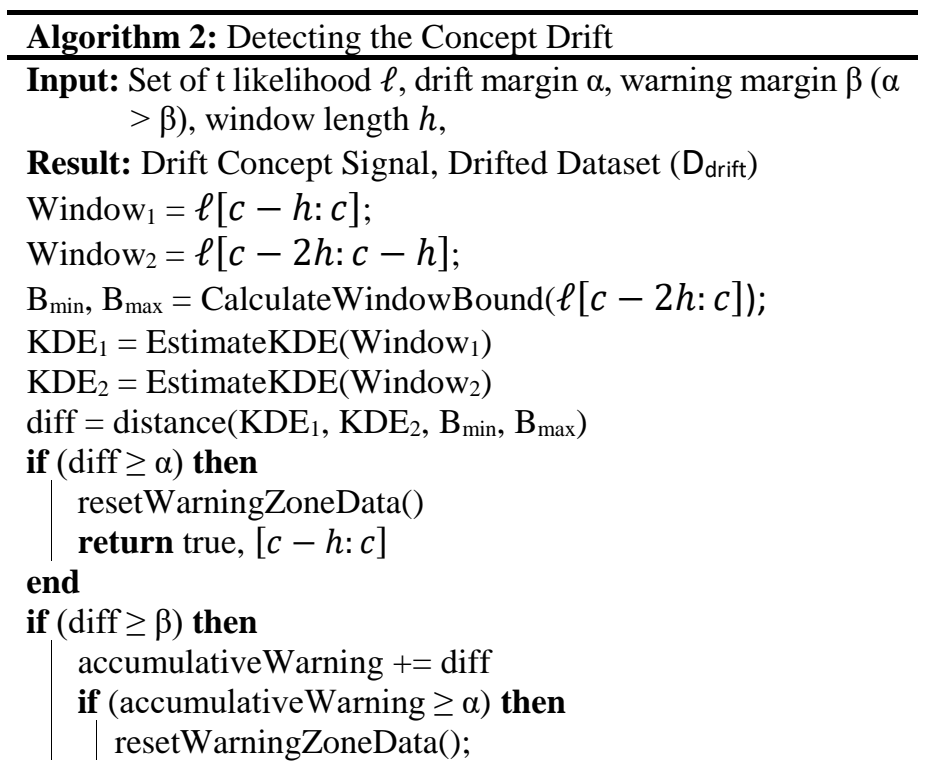




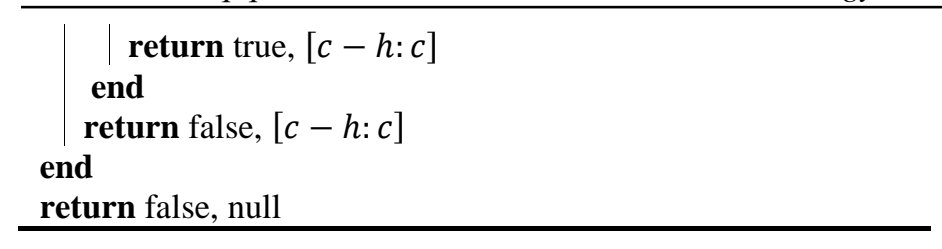

\subsection{Model Adaptation}

Model adaptation aims to revise the current model upon newly incoming data that might contain new concepts or concept changes. The result of this adaptation is an adapted weighted mixture component that respects the original mixture.

The model adaptation method starts by training a new model $M_{\text {drift }}$ from data drifts $D_{\text {drift }}$ using Algorithm 1, and then combining the existing model $\mathcal{M}$. Consequently, the newly adapted model $\mathcal{M}$ accommodates the new concept represented by the components in $\mathcal{M}_{\text {drift. }}$ The next step is to calculate the pairwise distance between the components in $\mathcal{M}$ using $\mathrm{KL}$ discrimination. The $\mathrm{KL}$ discrimination formula (Eq. (8)) enables us to set an upper bound on the discrimination of the mixture before and after the merging process. According to this formula, components with low weights means close to their variances, and similar covariance matrices are selected for merging. When two components are merged, the moment-preserving merging method [25] is used to preserve the mean and the covariance of the overall mixture (Eqs. (4)-(6)). Figure 4 illustrates the CMGM adaptation process.

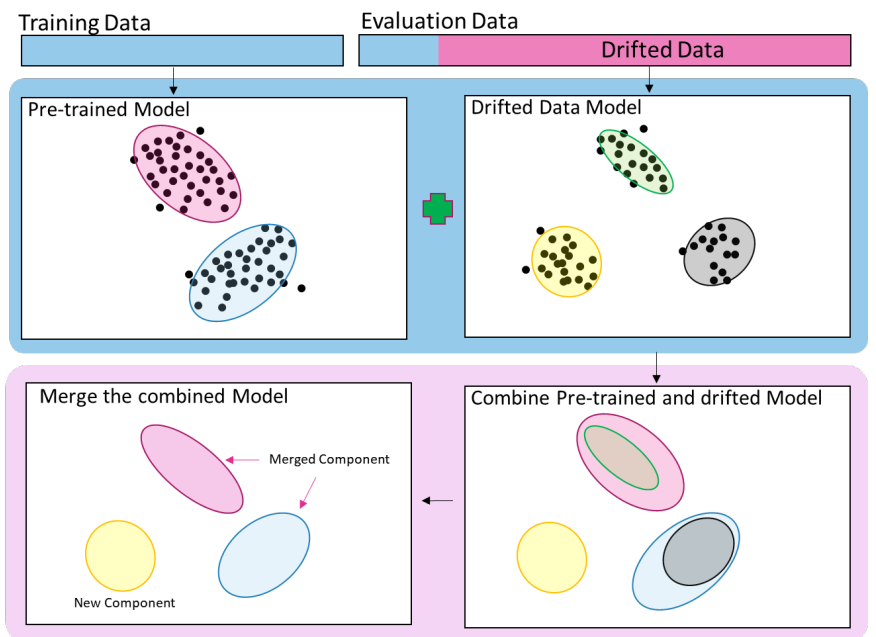

Figure 4: Illustration of the combine-merge Gaussian mixture model (CMGMM) adaptation process

As a result, the reduction process generates a set of merged models $\mathcal{M}_{\text {merge. }}$ To select the best $\mathcal{M}_{\text {merge }}$ model, the accumulative BIC is computed by combining sampling data from $\mathcal{M}_{\text {curr. }} \mathrm{D}_{\text {drift }}$ then computes the BIC value using Eq. (10). The smaller the value of the accumulative BIC, the better the newly adapted model.

Based on [7] and [11], the CMGMM tends to increase the number of components because it combines and merges them. This mechanism leads to an overfitting problem because the adaptation frequency increases due to the sensitive KD3 hyperparameter.

To maintain the compactness of the CMGMM and avoid overfitting, we design a strategy to merge statistically equivalent components into one component, then prune the inactive components. The inactive components are identified by the proximity of the ratio of $w^{2}$ and $\mathrm{P}^{2}$ of the merged component to zero. In practice, components with $w$ that are very close to zero are ignored by the model, whereas those with a large covariance tend to overlap with other components. Algorithm 3 presents in detail the steps of the proposed CMGMM-based method.

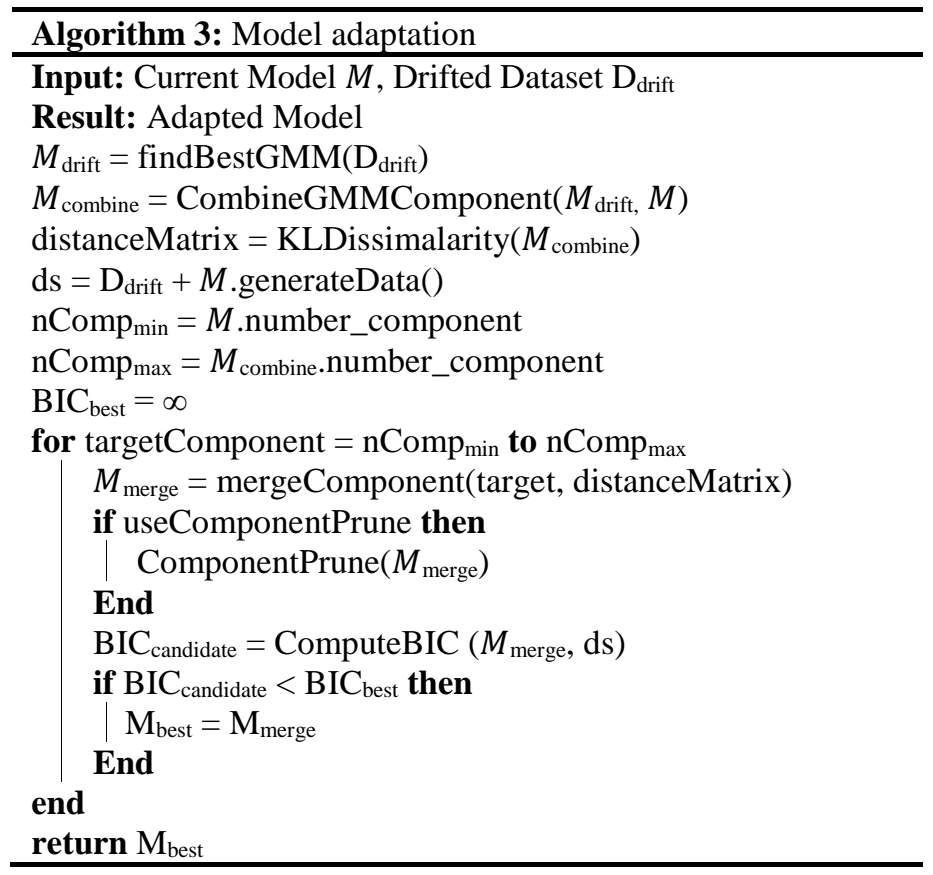

\section{Experiment}

This section provides information about the datasets and experimental setup used in this study to train, optimize, and evaluate the proposed method.

\subsection{Datasets}

We used three types of datasets in this experiment, that is, training, optimization, and evaluation. The training dataset consisted of audio signals extracted with a 10-seconds window from 15 scenes in the TUT Acoustic Scenes 2017 [26] and TAU Urban Acoustic Scenes 2019 datasets [26]. The scenes were home, airport, beach, office, cafe, grocery store, bus, tram, metro, city center, residential area, street pedestrian, and shopping mall.

To simulate the concept drift in the datasets, the optimization and evaluation datasets were generated by overlay new additional event sounds from and UrbanSound8K datasets [27] and the BBC Sound Class Library [28]. When the sounds were added, the numbers of additions (1-10), positions in the time axis (0-9000 $\mathrm{ms})$, and loudness $(-20,0)$ of the sounds were randomly changed at random. In total, the dataset had 46 new event classes and 371 added event sounds.

We generated four concept drift types with three scenarios. The four types of concept drift are as follows:

- abrupt concept drift (AB), where ongoing concepts are replaced with new concepts at a particular time;

- gradual concept drift (GR), where new concepts are added to an ongoing concept at a particular time; 
- $\quad$ recurring concept drift type 1 (R1), where an ongoing concept is replaced at a particular time with concepts that previously appeared; and

- $\quad$ recurring concept drift type 2 (R2), where concepts that previously appeared are added to an ongoing concept at a particular time.

Figure 5 illustrates the scenario generation. The three scenarios were designed to have different data distribution complexities. The scenario details are described below:

- Scenario 1 (Sc1): A unique event sounds from a specific event sounds is repeatedly introduced with a random number of times, gain, and timing. For example, in the airport scene, unique sounds representing the airplane sound, crowd background, and construction site are overlaid with a random number of times (1-10), position (0-9000 ms), and loudness $(-20,0)$.

- Scenario 2 (Sc2): Several event sounds are randomly selected from a set of the same sound labels in Sc1 and added using the same rule as Sc1.

- $\quad$ Scenario3 (Sc3): This scenario differs from Sc1 and Sc2 in that event sounds coexist among scenes. For example, a set of rain sounds exists in other scenes (e.g., beach, city center, and forest paths). The methods of selection and addition are the same as those in Sc2.

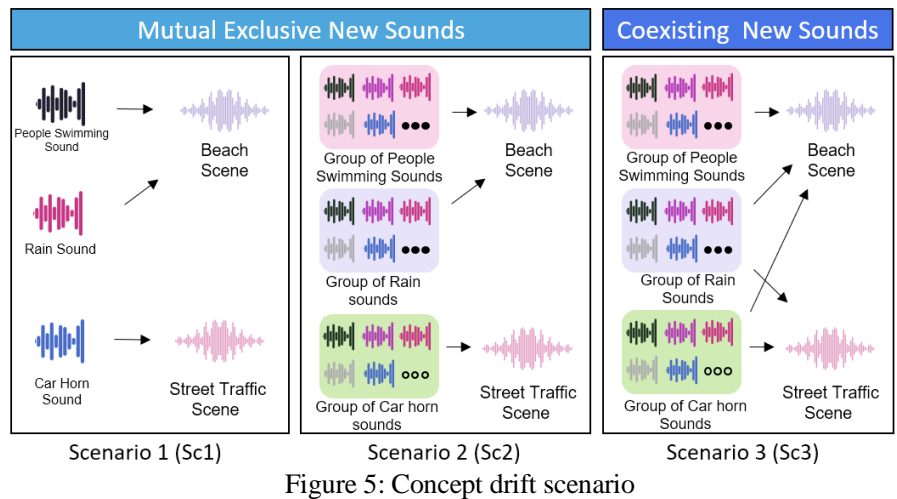

Table 1 shows a list of event sounds that appear at scene types in every concept drift scenario. The mutually exclusive event sounds appear in all scenarios, but coexisting sounds only appear for Sc3.

Table 1: Setting of the novel sounds in scene audio for Sc1, Sc2, and Sc3

\begin{tabular}{lll}
\hline Scene & \multicolumn{1}{c}{$\begin{array}{c}\text { Mutually Exclusive } \\
\text { Sounds in Sc1, Sc2, and } \\
\text { Sc3 }\end{array}$} & $\begin{array}{c}\text { Additional Coexisting } \\
\text { Sounds in Sc3 }\end{array}$ \\
\hline Airport & $\begin{array}{l}\text { Helicopter, crowd, and } \\
\text { construction site }\end{array}$ & $\begin{array}{l}\text { Airplane, footsteps, and } \\
\text { children playing }\end{array}$ \\
\hline Beach & $\begin{array}{l}\text { People swimming, footsteps } \\
\text { on the sand, and rain }\end{array}$ & $\begin{array}{l}\text { Teenage crowd, dog, and } \\
\text { birds }\end{array}$ \\
\hline Bus & Car horn, engine, and city & $\begin{array}{l}\text { Kitchenware, phone } \\
\text { ringing, children playing, } \\
\text { and teenage crowd }\end{array}$ \\
\hline
\end{tabular}

${ }^{2}$ https://bit.ly/CMGMM_Dataset

www.astesj.com

\begin{tabular}{|c|c|c|}
\hline $\begin{array}{l}\text { Café } \\
\text { /restaurant }\end{array}$ & $\begin{array}{l}\text { Washing machine, food } \\
\text { mixer, and kitchenware }\end{array}$ & $\begin{array}{l}\text { Phone ringing, children } \\
\text { playing, and teenage } \\
\text { crowd }\end{array}$ \\
\hline City center & $\begin{array}{l}\text { Sound of bird, ambulance, } \\
\text { and wind }\end{array}$ & $\begin{array}{l}\text { Footsteps, phone ringing, } \\
\text { and children playing }\end{array}$ \\
\hline $\begin{array}{l}\text { Grocery } \\
\text { store }\end{array}$ & $\begin{array}{l}\text { Footsteps, children playing, } \\
\text { and shopping cart }\end{array}$ & $\begin{array}{l}\text { Vacuum cleaner, phone } \\
\text { ringing, and footstep }\end{array}$ \\
\hline Home & $\begin{array}{l}\text { Frying, door, and vacuum } \\
\text { cleaner }\end{array}$ & Clock and phone ringing \\
\hline $\begin{array}{l}\text { Metro } \\
\text { station }\end{array}$ & Siren, road car, and thunder & $\begin{array}{l}\text { Footsteps, crowd, and } \\
\text { wind }\end{array}$ \\
\hline Office & $\begin{array}{l}\text { Typing, phone ringing, and } \\
\text { sneeze }\end{array}$ & $\begin{array}{l}\text { Broom, camera, and } \\
\text { footsteps }\end{array}$ \\
\hline $\begin{array}{l}\text { Public } \\
\text { square }\end{array}$ & $\begin{array}{l}\text { People running, music, and } \\
\text { airplane }\end{array}$ & $\begin{array}{l}\text { Birds, rain, and teenagers } \\
\text { talking }\end{array}$ \\
\hline $\begin{array}{l}\text { Residential } \\
\text { area }\end{array}$ & Wind, camera, and cat & Birds, sneeze, and clock \\
\hline $\begin{array}{l}\text { Shopping } \\
\text { mall }\end{array}$ & $\begin{array}{l}\text { Clock, camera, and teenage } \\
\text { crowd }\end{array}$ & $\begin{array}{l}\text { Children playing, phone } \\
\text { ringing, dog }\end{array}$ \\
\hline $\begin{array}{l}\text { Street } \\
\text { pedestrian }\end{array}$ & Dog, bicycle, and bird & $\begin{array}{l}\text { Footsteps and children } \\
\text { playing }\end{array}$ \\
\hline Street traffic & Motorcycle, horn, and train & Siren, airplane, and bell \\
\hline Tram & $\begin{array}{l}\text { Coughing, bell, and } \\
\text { footsteps on the pavement }\end{array}$ & $\begin{array}{l}\text { Teenage crowd and } \\
\text { children playing }\end{array}$ \\
\hline
\end{tabular}

Finally, we have one training dataset, four optimization datasets, and 12 evaluation datasets in this experiment. Each training dataset contained 3,000 scene audio, while the optimization and evaluation dataset contained 15,000 scene audio. The datasets are available in our repository ${ }^{2}$.

\subsection{Experimental Setup}

The CMGMM accuracy was evaluated under four concept drift types in three scenarios (i.e., Sc1, Sc2, and Sc3). The evaluations are performed using the two following approaches:

- Active CMGMM adaptation: In this approach, the CMGMM actively detects the concept drift using a certain method and only adapts the model when the concept drift is detected. In this study, we compared KD3 to ADWIN [9], HDDMA, HDDMW [10], and KSWIN [18].

- Passive CMGMM adaptation: In this approach, the CMGMM adapts as soon as a particular datum is received without requiring the explicit prior detection of the concept drift. Several adaptation cycle sizes were tested, that is, 25, 50, 100, 150 , and 200.

\section{Experiment Result}

The experimental result of the proposed method are presented herein.

\subsection{Hyperparameter Optimization}

The first step in this experiment is the systematic optimization of the KD3 hyperparameter. We used the grid-search method using a combination of hyperparameters $\alpha$ from 0.1 to $0.001, \beta$ from 0.0001 to 0.000001 , and $\mathrm{h}$ from 45 to 300 . We prepared a particular

. 
dataset for the KD3 hyperparameter optimization in four types of concept drift.

In [11], we reported that hyperparameters $\beta$ and $\mathrm{f}$ did not have a significant effect on accuracy. Therefore, during the initial step, we observed the performance change according to $\beta$ and $\mathrm{h}$. Figure 6 shows the average accuracy in all concept drift types according to hyperparameters $\beta$ and $h$. In this experiment, the best $\beta$ and $h$ were set at 0.0001 and 45 , respectively.

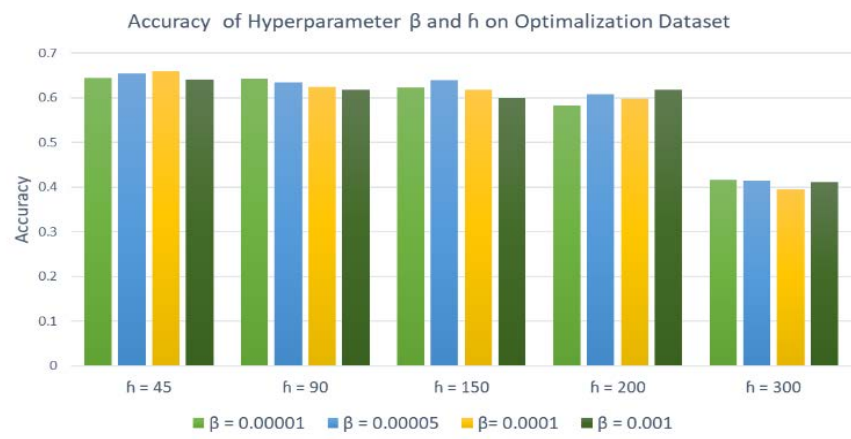

Figure 6: Result of hyperparameters $\beta$ and $\mathrm{f}$ in four types of concept drift

Table 2 lists the experimental results of $\alpha$ in the optimization dataset in four types of concept drift. Based on this experiment, every concept drift type has its respective hyperparameter $\alpha$ according to the concept drift characteristics. AB and GR have similar characteristics. There are no repeating concepts in the future; hence, a more sensitive concept drift detector than R1 and $\mathrm{R} 2$ is required.

Table 2: KD3 hyperparameter optimization result

\begin{tabular}{cccccc}
\hline \multirow{2}{*}{$\begin{array}{c}\text { Concept } \\
\text { Drift Types }\end{array}$} & \multicolumn{5}{c}{ Hyperparameter $\boldsymbol{\alpha}(\boldsymbol{\beta}=\mathbf{0 . 0 0 1}, \boldsymbol{h}=\mathbf{4 5})$} \\
\cline { 2 - 6 } & $\boldsymbol{\alpha}=\mathbf{0 . 1}$ & $\boldsymbol{\alpha}=\mathbf{0 . 0 5}$ & $\boldsymbol{\alpha}=\mathbf{0 . 0 1}$ & $\boldsymbol{\alpha}=\mathbf{0 . 0 0 5}$ & $\boldsymbol{\alpha}=\mathbf{0 . 0 0 1}$ \\
\hline $\mathrm{AB}$ & 0.6568 & 0.7113 & 0.7069 & $\mathbf{0 . 7 1 3 7}$ & 0.7066 \\
\hline $\mathrm{GR}$ & 0.6007 & 0.6173 & $\mathbf{0 . 7 0 5 0}$ & 0.6922 & 0.7002 \\
\hline $\mathrm{R} 1$ & $\mathbf{0 . 7 3 3 2}$ & 0.7232 & 0.7158 & 0.7054 & 0.6927 \\
\hline $\mathrm{R} 2$ & $\mathbf{0 . 7 2 6 8}$ & 0.7133 & 0.7228 & 0.7090 & 0.6823 \\
\hline Overall & 0.6793 & 0.6912 & $\mathbf{0 . 7 1 2 6}$ & 0.7050 & 0.6950 \\
\hline
\end{tabular}

In $\mathrm{AB}$ and $\mathrm{GR}$, a sensitive hyperparameter $\alpha$ accelerated the update frequency. In the experimental result for these types of concept drifts, a high adaptation frequency reduced the loss received. However, a less-sensitive hyperparameter showed a better result in recurring concept drifts, where an old concept reappears in the future. A less-sensitive hyperparameter $\alpha$ provided the model with longer data compared to the sensitive hyperparameter.

We also selected the overall hyperparameter setting based on this experiment. The overall hyperparameter was selected from the best average performance of the hyperparameter optimization $(\alpha=$ $0.01, \beta=0.001$, and $h=45$ ). We used this hyperparameter for further CMGMM and KD3 evaluations.

\subsection{Active Combine-Merge Gaussian Mixture Model (CMGMM) Adaptation Result}

Table 3 presents the experimental results of the active CMGMM adaptation. In general, the model performance without a concept drift detector is low in all concept drift types and scenarios. The adaptations of the CMGMM on R1 and R2 showed better accuracy than those on $A B$ and GR. On average, $A B$ exhibited the lowest accuracy, while R2 showed the highest accuracy. This high accuracy on recurring was caused by the CMGMM being designed to preserve the old concept, even though the new concept is adapted in the model. Thus, the model can recognize the previously learned concept if it is repeated in the future.

The CMGMM experiment result depicted that KD3 outperformed other combinations in two of the four concept drift types in GR and R2. Meanwhile, ADWIN showed the best accuracy in AB. KSWIN demonstrated the best accuracy in R1, whereas HDDM was unsuitable for this case. Despite getting the highest overall accuracy score, the combination of the CMGMM and KD3 needed more frequent adaptations than ADWIN and KSWIN. In contrast, both HDDM-based methods showed worse performances compared to all others. HDDMA overdetected the concept drift in all concept drift types for more than 3000 times in GR.

The abovementioned results illustrated that the concept drift detector plays a vital role in the concept drift adaptation. The model performance decreased over time if the drift detector failed to detect or delay detecting or over detecting the concept drift.

\section{Performance of the combine-merge Gaussian mixture model (CMGMM) and kernel density drift detection (KD3)}

KD3 showed the best average accuracy of 0.6983 compared to ADWIN, KSWIN, and HDMM with 209 adaptations. This combination also showed its best results on R2 with 0.7321 accuracy, followed by R1 with 0.7373 accuracy, GR with 0.6999 accuracy, and $\mathrm{AB}$ with accuracy 0.6469. Furthermore, this combination was the most stable in all scenarios. The maximum performance decrements in $\mathrm{AB}, \mathrm{GR}, \mathrm{R} 1$, and R2 were $1.38 \%$, $1.2 \%, 1.34 \%$, and $0.94 \%$, respectively.

Despite achieving a good performance in all concept drift types, the number of concept drifts detected in this combination was higher than ADWIN and KSWIN. The most significant number of adaptations occurred in AB. The disadvantage of a high number of adaptations is the higher computation time required to finish the task and possible overfitting. In this case, the higher numbers of adaptations in $\mathrm{AB}$ and GR are obtained because the concept constantly changes over time, and the learned concept becomes obsolete in the future; hence, the higher the adaptation, the better the performance.

\section{Performance of the combine-merge Gaussian mixture model (CMGMM) and ADWIN}

In general, the combination of CMGMM and ADWIN showed a good performance in every concept drift type, especially on the abrupt datasets, where this combination showed its best performance. The overall accuracy was 0.6371 with 83 times of adaptation. The overall accuracies of this combination in AB, GR, R1, and R2 were $0.6369,0.6475,0.6912$, and 0.7169 , respectively. Furthermore, this combination had the advantage of a small number of adaptations in all concept drift types. Hence, ADWIN showed an effective performance in using resources and had a reasonably good performance. This combination performed very well on Sc1, but showed a performance drop in Sc2 and Sc3. For 
Table 3: Experiment result of the CMGMM with the concept drift detector

\begin{tabular}{|c|c|c|c|c|c|c|c|c|c|c|c|c|c|}
\hline \multirow{2}{*}{\multicolumn{2}{|c|}{ Concept Drift Detector }} & \multicolumn{4}{|c|}{ Accuracy } & \multicolumn{4}{|c|}{ F1 } & \multicolumn{4}{|c|}{$\begin{array}{c}\text { Number of Concept Drift } \\
\text { Detection }\end{array}$} \\
\hline & & Sc1 & Sc2 & Sc3 & Overall & Sc1 & Sc2 & Sc3 & Overall & Sc1 & Sc2 & Sc3 & Overall \\
\hline \multirow{6}{*}{$\mathrm{AB}$} & ADWIN & 0.6989 & 0.6525 & 0.6214 & 99 & 0.713 & 0.6495 & 0.6353 & $\overline{99}$ & 83 & 89 & 85 & 85 \\
\hline & HDDM_A & 0.4157 & 0.4476 & & & 0.4586 & 0.4804 & & & 3287 & 3350 & 3359 & 3332 \\
\hline & HDDM_W & 041 & 0.4455 & & & & 0.4902 & 0.5004 & & & 413 & 409 & 437 \\
\hline & KD3* & 469 & 0.6359 & & 36 & 0.6476 & 0.6467 & 0.6395 & 3446 & 207 & 236 & 220 & 221 \\
\hline & KSWII & 611 & 0.6241 & 5 & & 0.6722 & 0.6369 & 0.6411 & 6501 & 132 & 121 & 123 & 125 \\
\hline & Wi & 1121 & 0.4054 & 4095 & 90 & 0.4235 & 0.4125 & 0.4095 & 4151 & & & & \\
\hline \multirow{6}{*}{ GR } & & 723 & 0.6341 & 6363 & 75 & 0.6845 & 0.6506 & 0.6478 & 6609 & 92 & 81 & 83 & 85 \\
\hline & & 134 & 0.4463 & 4311 & 302 & 0.4580 & 0.4946 & 0.4701 & 42 & 306 & 3308 & 3265 & 3293 \\
\hline & HDI & 0.4131 & 0.4497 & 0.4544 & 0.439 & 0.4524 & 0.487 & 0.4816 & 36 & 0 & 409 & 401 & 270 \\
\hline & KD3 & 0.6999 & 0.6942 & 0.6879 & 0.694 & 0.7044 & 0.7004 & 0.6867 & & 190 & 218 & 221 & 209 \\
\hline & KSI & 532 & 0.6241 & 0.618 & 0.6322 & 0.6631 & 0.6326 & 0.6204 & & 129 & 125 & 107 & 120 \\
\hline & With & 0.3554 & 0.3524 & 0.3489 & 0.3522 & 0.3571 & 0.3542 & 0.3501 & 0.3538 & & & & \\
\hline \multirow{6}{*}{ R1 } & $\mathrm{ADW}$ & 0.7222 & 0.6948 & 0.6568 & & 0.7393 & 0.6981 & 0.6333 & & 78 & 83 & 87 & 82 \\
\hline & $\mathrm{H}$ & 4721 & 0.5204 & $0.4 \varepsilon$ & 94 & 0.5206 & 0.5566 & 0.5392 & & 154 & 3258 & 3201 & 3204 \\
\hline & HDDM & 0.4847 & 0.4815 & 0.5068 & 0.491 & 0.5305 & 0.5215 & 0.5357 & 92 & 0 & 662 & 647 & 436 \\
\hline & KD3* & 0.7373 & 0.7334 & 0.7239 & 0.7315 & 0.7389 & 0.7341 & 0.7247 & 25 & 208 & 201 & 204 & 204 \\
\hline & KSWI & 0.7818 & 0.7351 & 0.7357 & 0.7508 & 0.7876 & 0.7404 & 0.7416 & 65 & 142 & 135 & 126 & 134 \\
\hline & Without I & 0.4512 & 0.4458 & 0.4257 & 0.4409 & 0.4512 & 0.4458 & 0.4257 & 0.4409 & & & & \\
\hline \multirow{6}{*}{$\mathrm{R} 2$} & & & & & & & & & & & 86 & & 81 \\
\hline & & & 0.6 & & & & & & & 3052 & 2957 & ב & 3008 \\
\hline & HD & & & & & & & & & 759 & 604 & 543 & 635 \\
\hline & KD & & & & & & & & & & 207 & 20 & 202 \\
\hline & & & & & & & & & & 156 & 143 & & 146 \\
\hline & Without D & 0.4254 & 0.4205 & 0.3985 & 0.4148 & 0.4279 & 0.4198 & 0.3968 & 0.4148 & & & & \\
\hline \multirow{6}{*}{$\begin{array}{l}\overline{\bar{\sigma}} \\
\bar{\Xi} \\
\bar{\partial}\end{array}$} & ADWIN & 0.7072 & 0.6720 & 0.6559 & 0.6731 & 0.7191 & 0.6778 & 0.6581 & 0.6835 & 02 & 84 & 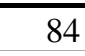 & 83 \\
\hline & HDDM_ & 0.4733 & 0.5044 & 0.4953 & 0.4910 & 0.5185 & 0.5428 & 0.5380 & 5331 & 3199 & 3218 & 3210 & 3209 \\
\hline & HDDM_W & 0.4702 & 0.4941 & 0.5008 & 0.4883 & 0.5170 & 0.5346 & 0.5458 & & 312 & 522 & 500 & 444 \\
\hline & KD3* & 7041 & 0.6990 & 0.6919 & 0.6983 & 0.7065 & 0.7031 & 0.6932 & & 200 & 215 & 212 & 209 \\
\hline & & 969 & 0.6699 & 0.66 & 0.6757 & 0.7051 & 0.6768 & 0.6710 & 0.6843 & 139 & 131 & 124 & 131 \\
\hline & Without Detector & 0.4110 & 0.4060 & 0.3956 & 0.4042 & 0.4149 & 0.4080 & 0.3955 & 0.4061 & & & & \\
\hline
\end{tabular}

(*) Proposed method

example, the accuracies of AB, GR, R1, and R2 decreased in Sc3 by $4.64 \%, 3.82 \%, 2.74 \%$, and $287 \%$, respectively.

\section{Performance of the combine-merge Gaussian mixture model (CMGMM) and Hoeffding's bounds-based method (HDDM)}

In this experiment, both Hoeffding's inequality-based algorithms showed underperformance results for all concept drift types. Both algorithms were less effective in detecting the concept drift in this case. The overall accuracies of HDDMA in AB, GR, $\mathrm{R} 1$, and R2 were $0.4326,0.4302,0.494$, and 0.607 , respectively. The number of HDDMA adaptations exceeded 3000 times of adaptation. This high adaptation process was ineffective because the amount of trained data for each adaptation was too small. This condition led to an overfitting and decreased the model performance.

HDDM W also experienced the same problem. In some cases, HDDMA failed to detect the drift concepts, such as GR, R1, and $\mathrm{R} 2$ in Sc1. The overall accuracies of this HDDMW in AB, GR, R1, and R2 were $0.4347,0.439,0.491$, and 0.5886 , respectively.

\section{Performance of the combine-merge Gaussian mixture model} (CMGMM) and KSWIN

The combination of CMGMM and KSWIN showed the best performance in R1, with an overall accuracy of 0.750 with 134 adaptations. The accuracies of this combination in AB, GR, R1, and R2 were $0.6317,0.6322,0.7508$, and 0.6882 , respectively. On average, KSWIN required eight to nine adaptations per scene in all dataset types. This algorithm seems able to detect occurring changes in data and supports the concept drift handling process with good indicators at a given time.

\subsection{Passive Combine-Merge Gaussian Mixture Model (CMGMM) Adaptation Result}

Table 4 lists the experimental results of the passive CMGMM adaptation. The best performance in AB, GR, and R1 was obtained with a cycle size of 50, and that in R2 was obtained with a cycle size of 100 . The best accuracies of AB, GR, R1, and R2 were $0.7152,0.7139,0.7323$, and 0.7155 , respectively. 
I. Daqiqil ID et al./Advances in Science, Technology and Engineering Systems Journal Vol. 6, No. 5, 167-176 (2021)

Table 4: The experiment result of CMGMM without concept drift detector

\begin{tabular}{|c|c|c|c|c|c|}
\hline \multirow{2}{*}{$\begin{array}{c}\text { Concept } \\
\text { Drift } \\
\text { Types }\end{array}$} & \multirow{2}{*}{$\begin{array}{c}\text { Cycle } \\
\text { Size }\end{array}$} & \multicolumn{4}{|c|}{ Accuracy } \\
\hline & & Sc1 & Sc2 & Sc3 & Average \\
\hline \multirow{5}{*}{$\mathrm{AB}$} & 25 & 0.6290 & 0.6236 & 0.6256 & 0.6260 \\
\hline & 50 & 0.7122 & 0.7159 & 0.7177 & 0.7152 \\
\hline & 100 & 0.6285 & 0.5925 & 0.5955 & 0.6055 \\
\hline & 150 & 0.6361 & 0.5776 & 0.5851 & 0.5996 \\
\hline & 200 & 0.5580 & 0.5059 & 0.5119 & 0.5252 \\
\hline \multirow{5}{*}{ GR } & 25 & 0.6294 & 0.6232 & 0.6004 & 0.6176 \\
\hline & 50 & 0.7133 & 0.7169 & 0.7115 & 0.7139 \\
\hline & 100 & 0.6173 & 0.5887 & 0.6089 & 0.6049 \\
\hline & 150 & 0.6371 & 0.5818 & 0.5797 & 0.5995 \\
\hline & 200 & 0.5334 & 0.5094 & 0.5076 & 0.5168 \\
\hline \multirow{5}{*}{ R1 } & 25 & 0.6186 & 0.5799 & 0.5608 & 0.5864 \\
\hline & 50 & 0.7235 & 0.7320 & 0.7416 & 0.7323 \\
\hline & 100 & 0.7211 & 0.7105 & 0.6988 & 0.7101 \\
\hline & 150 & 0.7332 & 0.7086 & 0.7120 & 0.7179 \\
\hline & 200 & 0.6639 & 0.7018 & 0.7146 & 0.6934 \\
\hline \multirow{5}{*}{$\mathrm{R} 2$} & 25 & 0.5133 & 0.5444 & 0.5669 & 0.5415 \\
\hline & 50 & 0.7396 & 0.6991 & 0.7011 & 0.7132 \\
\hline & 100 & 0.7431 & 0.7012 & 0.7023 & 0.7155 \\
\hline & 150 & 0.6865 & 0.6639 & 0.6803 & 0.6769 \\
\hline & 200 & 0.6502 & 0.6011 & 0.6089 & 0.6200 \\
\hline
\end{tabular}

Similar to active adaptation, R1 and R2 showed good performances compared to $\mathrm{AB}$ and $\mathrm{GR}$, but better performances in passive adaptation. Although R1 exhibited the best adaptation at cycle size 50, it also showed good result at cycle sizes 100 and 150. If you consider the time and the computing resources used, then cycle sizes 100 and 150 are recommended.

In passive adaptation, the cycle size is vital in achieving a good performance. This cycle size determines the adequacy of the data for adaptation. If the cycle size is too short, the number of data adapted is small, leading to overfitting problems.

\subsection{Suggestions and Limitations}

The experiment results showed that the combination of CMGMM and KD3 has a higher number of adaptations compared to that of ADWIN and KSWIN due to the selection of KD3 hyperparameters that are sensitive to accommodating GR and AB. When the number of adaptations is increased, then in certain cases, such as GR and AB, the accuracy is improved, albeit with a higher computing cost. The advantage of KD3 compared to the other methods is that it could be applied in multi-dimensional probability distribution; hence, it is more flexible to apply in other models and cases.

In cases where the time or location of the concept drift can be predicted, the use of a passive adaptation strategy is more beneficial and has a lower computational cost than the active strategy. However, if the adaptation cycle is too far from the concept drift, then the model performance will decrease over time.

\section{Conclusion}

This paper presented KD3 hyperparameter optimization and the CMGMM performance evaluation in four types of concept drift. All concept drift types had optimum hyperparameter configurations. $\mathrm{AB}$ and GR showed similar patterns that required a smaller $\alpha$ value than the recurring concept drift. In this type of concept drift, concepts continuously appear; hence, a sensitive drift detector is needed to update the model early or have a highfrequency model adaptation. However, in recurring concepts, the new concept may be repeated in the future; thus, a lower-frequency adaptation shows a better performance.

The evaluation results demonstrated that the proposed algorithms work well in detecting and adapting to four types of concept drift and three scenarios. Overall, the CMGMM works better in R1 and R2 than in AB and GR because it is designed to preserve old concepts to preserve previously learned knowledge. Component pruning is only performed on components with a minimal impact on the prediction.

In the active adaptation strategy, the proposed combined method of CMGMM and KD3 outperformed two of four other combination methods in GR and R1. These methods showed the most stable performance among Sc1, Sc2, and Sc3 in all concept drift types. Furthermore, ADWIN showed the best results on AB. This algorithm is efficient in computing resources, but less stable in more challenging scenarios, such as Sc2 and Sc3. KSWIN showed the best results in recurring drift and good performance for all concept drift types. HDDM overdetected or failed to detect the concept drift and was not suitable in this case.

In the passive adaptation strategy, a short or long adaptation cycle could reduce the performance, and a short cycle size makes the adaptation less effective. A short cycle could disrupt the model component because the model is trained with insufficient data, leading to an underfitting problem.

In $\mathrm{AB}$ and $\mathrm{GR}$, the model requires a high-frequency adaptation to maintain the performance; therefore, sensitive hyperparameters or a short cycle size is required. Based on the experimental results, the passive method is more suitable in these concept drift types. However, the computational costs are higher than those of the active method. Furthermore, in recurring drift scenarios, a lesssensitive hyperparameter, or a moderate cycle size is needed. The active method is recommended for recurring drift types considering the computation cost and better accuracy in experiments.

As part of our future work, we plan to improve the proposed KD3 concept drift detection algorithm to achieve adaptive weight, reduce the kernel density computation, and optimize the number of data points considered when detecting the concept drift and decreasing the computation time.

\section{Conflict of Interest}

The authors declare no conflict of interest.

\section{Acknowledgment}

This work was supported by JSPS KAKENHI Grant Number JP20K12079.

\section{References}

[1] R. Elwell, R. Polikar, "Incremental Learning of Concept Drift in Nonstationary Environments," IEEE Transactions on Neural Networks, 22(10), 1517-1531, 2011, doi:10.1109/TNN.2011.2160459.

[2] S. Ntalampiras, "Automatic analysis of audiostreams in the concept drift environment," in 2016 IEEE 26th International Workshop on Machine Learning for Signal Processing (MLSP), IEEE, 2016, doi:10.1109/MLSP.2016.7738905. 
[3] J. Gama, I. Žliobaitė, A. Bifet, M. Pechenizkiy, A. Bouchachia, "A Survey on Concept Drift Adaptation,” ACM Computing Surveys, 46(4), 1-37, 2014, doi:10.1145/2523813.

[4] T.R. Hoens, R. Polikar, N. V. Chawla, "Learning from Streaming Data with Concept Drift and Imbalance: An Overview," Progress in Artificial Intelligence, 1(1), 89-101, 2012, doi:10.1007/s13748-011-0008-0.

[5] I. Žliobaitè, "Learning under Concept Drift: an Overview," 1-36, 2010, doi:10.1002/sam.

[6] C. Chen, C. Wang, J. Hou, M. Qi, J. Dai, Y. Zhang, P. Zhang, “Improving Accuracy of Evolving GMM under GPGPU-Friendly Block-Evolutionary Pattern,” International Journal of Pattern Recognition and Artificial Intelligence, 34(3), 1-34, 2020, doi:10.1142/S0218001420500068.

[7] I.D. Id, M. Abe, S. Hara, "Concept Drift Adaptation for Acoustic Scene Classifier based on Gaussian Mixture Model,” in IEEE Region 10 Annual International Conference, Proceedings/TENCON, IEEE, Osaka: 450-455, 2020, doi:10.1109/TENCON50793.2020.9293766.

[8] J.M. Acevedo-Valle, K. Trejo, C. Angulo, "Multivariate regression with incremental learning of Gaussian mixture models," Frontiers in Artificial Intelligence and Applications, 300, 196-205, 2017, doi:10.3233/978-161499-806-8-196.

[9] A. Bifet, R. Gavaldà, "Learning from Time-changing Data with Adaptive Windowing," Proceedings of the 7th SIAM International Conference on Data Mining, (April), 443-448, 2007, doi:10.1137/1.9781611972771.42.

[10] I. Frías-Blanco, J. Del Campo-Ávila, G. Ramos-Jiménez, R. Morales-Bueno, A. Ortiz-Díaz, Y. Caballero-Mota, “Online and Non-Parametric Drift Detection Methods Based on Hoeffding's Bounds," IEEE Transactions on Knowledge and Data Engineering, 27(3), 810-823, 2015, doi:10.1109/TKDE.2014.2345382.

[11] I.D. Id, M. Abe, S. Hara, "Evaluation of Concept Drift Adaptation for Acoustic Scene Classifier Based on Kernel Density Drift Detection and Combine Merge Gaussian Mixture Model,” in 2021 Spring meeting of the Acoustical Society of Japan, 2021.

[12] J. Montiel, J. Read, A. Bifet, T. Abdessalem, "Scikit-multiflow: A Multioutput Streaming Framework,” Journal of Machine Learning Research, 19, 1-5, 2018, doi:10.5555/3291125.3309634.

[13] G.I. Webb, R. Hyde, H. Cao, H.L. Nguyen, F. Petitjean, “Characterizing Concept Drift,” Data Mining and Knowledge Discovery, 30(4), 964-994, 2016, doi:10.1007/s10618-015-0448-4.

[14] T. Zhang, J. Liang, B. Ding, "Acoustic scene classification using deep CNN with fine-resolution feature,” Expert Systems with Applications, 143(1), 113067, 2020, doi:10.1016/j.eswa.2019.113067.

[15] A. Tsymbal, “The Problem of Concept Drift: Definitions and Related Work,” 2004.

[16] E.S. Page, “Continuous Inspection Schemes,” Biometrika, 41(1/2), 100, 1954, doi:10.2307/2333009.

[17] Y. Yuan, Z. Wang, W. Wang, "Unsupervised concept drift detection based on multi-scale slide windows," Ad Hoc Networks, 111, 102325, 2021, doi:10.1016/j.adhoc.2020.102325.

[18] C. Raab, M. Heusinger, F.M. Schleif, "Reactive Soft Prototype Computing for Concept Drift Streams,” Neurocomputing, 2020, doi:10.1016/j.neucom.2019.11.111.

[19] A.R. Runnalls, "Kullback-Leibler Approach to Gaussian Mixture Reduction,” IEEE Transactions on Aerospace and Electronic Systems, 43(3), 989-999, 2007, doi:10.1109/TAES.2007.4383588.

[20] A.P. Dempster, N.M. Laird, D.B. Rubin, "Maximum Likelihood from Incomplete Data via the EM Algorithm,” Journal of the Royal Statistical Society. Series B (Methodological), 39(1), 1-38, 1977.

[21] A.D.R. McQuarrie, C.-L. Tsai, Regression and Time Series Model Selection, WORLD SCIENTIFIC, 1998, doi:10.1142/3573.

[22] P. Pal Singh, “An Approach to Extract Feature using MFCC,” IOSR Journal of Engineering, 4(8), 21-25, 2014, doi:10.9790/3021-04812125.

[23] C. Fraley, "How Many Clusters? Which Clustering Method? Answers Via Model-Based Cluster Analysis,” The Computer Journal, 41(8), 578-588, 1998, doi:10.1093/comjnl/41.8.578.

[24] E. Parzen, “On Estimation of a Probability Density Function and Mode,” The Annals of Mathematical Statistics, 33(3), 1065-1076, 1962, doi:10.1214/aoms/1177704472.

[25] J.L. Williams, P.S. Maybeck, "Cost-function-based Gaussian mixture reduction for target tracking," Proceedings of the 6th International Conference on Information Fusion, 2, 1047-1054, 2003, doi:10.1109/ICIF.2003.177354.

[26] A. Mesaros, T. Heittola, T. Virtanen, TUT Acoustic scenes 2017, Development dataset, 2017, doi:10.5281/ZENODO.400515.

[27] J. Salamon, C. Jacoby, J.P. Bello, “A Dataset and Taxonomy for Urban Sound Research,” MM 2014 - Proceedings of the 2014 ACM Conference on

www.astesj.com
Multimedia, (November), 1041-1044, 2014, doi:10.1145/2647868.2655045.

[28] BBC, BBC Sound Effects, https://sound-effects.bbcrewind.co.uk/search, Mar. 2019. 